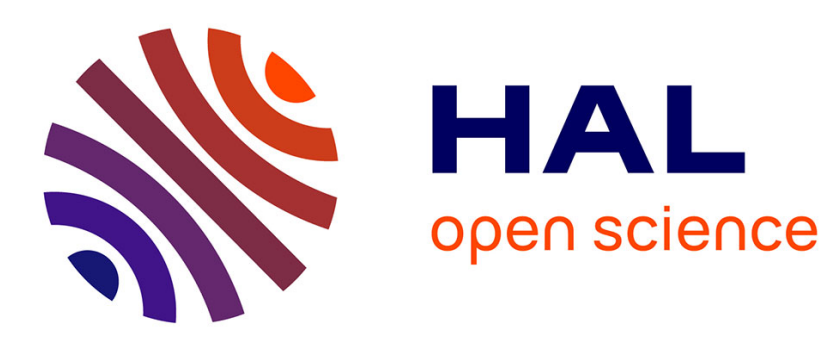

\title{
Sparse Approximations in Signal and Image Processing - EDITORIAL
}

\author{
Rémi Gribonval, Morten Nielsen
}

\section{To cite this version:}

Rémi Gribonval, Morten Nielsen. Sparse Approximations in Signal and Image Processing - EDITORIAL. Signal Processing, 2006, special issue on Sparse Approximations in Signal and Image Processing, 86 (3), pp.415-416. 10.1016/j.sigpro.2005.07.012 . inria-00544985

\section{HAL Id: inria-00544985 https://hal.inria.fr/inria-00544985}

Submitted on 20 Aug 2013

HAL is a multi-disciplinary open access archive for the deposit and dissemination of scientific research documents, whether they are published or not. The documents may come from teaching and research institutions in France or abroad, or from public or private research centers.
L'archive ouverte pluridisciplinaire HAL, est destinée au dépôt et à la diffusion de documents scientifiques de niveau recherche, publiés ou non, émanant des établissements d'enseignement et de recherche français ou étrangers, des laboratoires publics ou privés. 


\section{Guest Editorial \\ Sparse Approximations in Signal and Image Processing}

Sparse approximation techniques have been at the core of a rapidly evolving and very active area of research since the 1990s. Their most visible technological success has certainly been in the compression of high-dimensional data with wavelets. However, approximating a signal or an image with a sparse linear expansion from a possibly overcomplete dictionary of basis functions (called atoms) has turned out to be an extremely useful tool to solve many other signal processing problems, including blind source separation, feature extraction and classification, denoising, and detection, to name only a few. This special issue gives an overview of current developments with a collection of articles related to applications of sparse approximation and sparse coding techniques to images as well as audio and biomedical signals, new efficient implementations of greedy algorithms, performance analysis of greedy and convex optimization algorithms, as well as multichannel signal representations and source separation.

The first six papers investigate important issues related to the design -or the choice- of a dictionary adapted to a class of signals of interest, and to the implementation of efficient algorithms that take into account the special structure of the dictionaries at hand. In the paper by Plumbley et al., the main purpose is music signal transcription and representation which is performed through dictionary learning and sparse coding. Audio and image coding with multilayer dictionaries are considered in the papers by Vera-Candeas et al. and Peotta et al., the purpose being to encode efficiently both transients/edges and sinusoids/textures by combining elementary dictionaries into a larger one. With a special focus on computational complexity issues, Davies and Daudet also consider audio coding with a dictionary corresponding to a Modified Complex Lapped Transform seen as the union of a local cosine basis and a local sine basis. Similarly, for dictionaries of tight wavelet frames, Petukhov provides an efficient method to perform Orthogonal Matching Pursuit (also know as the orthogonal greedy algorithm). Finally, the paper by Andrle and Rebollo-Neira proposes a new refinement of Orthogonal Matching Pursuit which both improves the approximation performance for a given number of iterations and has a low computational complexity.

Another strong trend of research is represented in the following four papers, which are dedicated to the analysis of the performance of various sparse ap- 
proximation algorithms. Inspired by - and building upon - the seminal paper "Uncertainty Principles and Ideal Atomic Decompositions" by D. Donoho and $\mathrm{X}$. Huo, they analyze the connections between the output of Matching Pursuit, Basis Pursuit, or some other types of sparse approximation algorithms, and properties of ideal sparse representations/approximations of the analyzed input signal. The paper by Gribonval et al. provides a simple test to check $a$ posteriori whether the output of an arbitrary sparse approximation algorithm is nearly optimal, with respect to a large family of sparsity measures, and for various possible distortion measures. Donoho and Elad consider the stability of Basis Pursuit when a truely sparse input signal is corrupted by noise of small $\ell^{\infty}$ norm, with results both for general incoherent dictionaries and for unions of incoherent orthonormal bases. The first paper by Tsaig and Donoho is a heuristical and numerical study of the behaviour of Basis Pursuit when one deviates from the sufficient sparsity conditions under which Basis Pursuit always succeeds in recovering the truely sparsest representation of a signal. In their second paper, Tsaig and Donoho address the recently emerging topic of compressed sensing, where the core idea is to get accurate an reconstruction of a signal using very few (generally random) linear measurements, assuming that the signal has some sparse but unknown representation.

The final four papers of this issue are dedicated to the study of joint sparse approximation of several signals, with its application to blind source separation and multichannel signal representation. Tropp and his co-authors introduce and study both greedy algorithms (Matching Pursuit) and convex optimization techniques (similar to Basis Pursuit) in the context of simultaneous sparse approximation of several signals. In the paper of Theis and García, the idea of sparse component analysis -which combines dictionary learning and sparse representations- is applied to surface electromyograms and compared to other blind source separation techniques. Finally, Model and Zibulevsky propose a method to estimate directions of arrival and to reconstruct unknown sources from multisensor measurement, based on both "spatial" and "inner" sparsity of the emitted sources on time-frequency dictionaries.

The idea for this special issue arose from discussions with Pr. Pierre Vandergheynst. The Guest Editors would like to thank him and Murat Kunt, Editor in Chief of EURASIP Signal Processing, for providing the opportunity to prepare this special issue. Also, we thank all the authors who submitted manuscripts and all the referees for their hard work and conscientious refereeing.

R. Gribonval and M. Nielsen (Guest Editors)

\section{R. Gribonval}


IRISA, Campus de Beaulieu, 35042 Rennes Cedex, France

M. Nielsen mnielsen@math.aau.dk

Dept of Math. Sciences, Aalborg University, Fredrik Bajers Vej 7G, 9220 Aalborg East, Denmark 